\title{
Artículos
}

\section{Valoración del potencial turístico del paisaje en el municipio Isidro Fabela, México*}

Cómo citar este artículo: García-Romero, A., Gutiérrez-López, D. M., García-Sánchez, L. A., Méndez-Méndez, A., y Serrano de la Cruz SantosOlmo, M. A. (202I). Valoración del potencial turístico del paisaje en el municipio Isidro Fabela, MéxicoCuadernos de Desarrollo Rural, I8. https://doi.org/ı0.III44/Javeriana.cdrı8.vptp

Arturo García-Romero

Universidad Nacional Autónoma de México, México

ORCID: https://orcid.org/0000-0002-6339-1577

Dalia Margarita Gutiérrez-López ${ }^{\text {a }}$

Universidad Nacional Autónoma de México, México

gutierrezl_dalia@hotmail.com

ORCID: https://orcid.org/0000-0003-I499-0466

Laeticia Azucena García-Sánchez

Universidad Nacional Autónoma de México, México

ORCID: https://orcid.org/0000-0002-8986-8193

Alberto Méndez-Méndez

Universidad Nacional Autónoma de México, México

ORCID: https://orcid.org/0000-000I-89II-8I33

Manuel Antonio Serrano de la Cruz Santos-Olmo

Universidad de Castilla-La Mancha, España

ORCID: https://orcid.org/0000-0003-0I22-4877

DOI: https://doi.org/ıo.III44/Javeriana.cdri8.vptp

Recibido: 2I Octubre 2019 I Aceptado: OI Enero 202I I Publicación: 25 Agosto 202I

a Autora de correspondencia. Correo electrónico: gutierrezl_dalia@hotmail.com 


\title{
Resumen:
}

En el presente estudio, se aplica un método para evaluar el potencial turístico que tiene el paisaje, en un área rural próxima a la Ciudad de México. El análisis se basa en la aplicación de indicadores para valorar la calidad de los atractivos turísticos y así integrarla como valor añadido al potencial turístico de los paisajes. En la investigación, fueron identificados dos tipos de paisaje, cuyo potencial se fundamenta en la diversidad y la calidad de sus recursos biofísicos; asimismo, se identificaron veintidós atractivos turísticos, ocho de los cuales son de calidad media, claves para impulsar el turismo en un sector más amplio de la comunidad. Los resultados obtenidos destacan la importancia que tiene la relación entre paisaje y turismo, en especial, en la planificación de proyectos turísticos.

Palabras clave: calidad del paisaje, atractivo turístico, indicadores ambientales, planeación del turismo, turismo alternativo, desarrollo rural.

\section{Assessing the Tourism Potential of the Landscape in the Town Isidro Fabela, Mexico}

\begin{abstract}
:
This study provides a method to assess the tourism potential of the landscape in a rural area near Mexico City. The analysis is based on applying indicators for assessing the quality of tourism attractions and integrating it as an added value to the landscape tourism potential. This research identified two kinds of landscape whose potential lies in the diversity and quality of the biophysical resources. Additionally, 22 tourism attractions were identified, 8 of them are of medium quality and key to drive the tourism more widely among the community. The results obtained herein underline how important is the relationship between landscape and tourism, especially the planning of tourism projects.
\end{abstract}

Keywords: landscape quality, tourism attraction, environmental indicators, tourism planning, alternative tourism, rural development.

En las últimas décadas, el turismo se ha expandido al ámbito rural de los llamados países en desarrollo. Innumerables proyectos turísticos, vinculados en su base con las diversas modalidades del llamado turismo sustentable, el cual incluye al ecoturismo y al turismo rural, entre otros, han proliferado como una alternativa o complemento a los medios de vida tradicionales (Bucheli et al., 2009; Carneiro et al., 2015; Robín et al., 2015).

Una propuesta que desarrolla la planificación de este tipo de proyectos es la evaluación del potencial turístico del paisaje (Zuluaga, 2006; Dosso, 20II; Mikulec y Antoušková, 20II; Méndez-Méndez et al., 20I8; Salinas et al., 2019), la cual se define como el conjunto de condiciones naturales y culturales que permiten que el paisaje sea un incentivo en el desarrollo de las actividades turísticas (Picazo, 20I2; Santos-Pavón et al., 2016). El desarrollo de nuevas formas promueven un turismo cada vez más informado, responsable, justo y sostenible, lo que ha permitido considerar, en las evaluaciones del potencial turístico del paisaje, además del valor estético de los sitios, otros elementos importantes como el valor ecológico, educativo, espiritual, interpretativo e histórico, los cuales apuestan por la autenticidad y singularidad de los territorios (Torres, 2003; Tarroja, 2004; Meng y Uysal, 2008; Rivera y Rodríguez, 2012; Willson et al., 2013; Lane y Kastenholtz, 2015; Cebrián y García, 2016; Jerez y Serrano de la Cruz, 20ı6; Pié i Ninot et al., 2019).

$\mathrm{Al}$ ser el turismo una actividad compleja, es de especial interés del presente estudio sustentar una evaluación precisa y sistemática del potencial turístico del paisaje, a partir del análisis de indicadores (Serrano, 2008; Nogué et al., 2009). En general, la utilidad que tiene el análisis de los indicadores radica en que son suficientemente abiertos para evaluar una amplia gama de casos, al tiempo que son lo suficientemente puntuales para precisar las evaluaciones (Organization for Economic Co-operation and Development [OECD], 2003; Nogué et al., 2009 y 2019). En el ámbito latinoamericano, diversos estudios han utilizado indicadores ambientales para evaluar la 
identidad, la calidad o el atractivo visual del paisaje, lo que ha producido diagnósticos más precisos y veraces, en aras de mejorar la sostenibilidad de los proyectos turísticos en estudio (Serrano, 2008; Mearns, 20I2; Carneiro et al., 2015). Sin embargo, los casos en los que se lleva a cabo la aplicación continúan siendo escasos, particularmente, al considerar la compleja situación de la actividad turística en las áreas rurales.

Específicamente, en este estudio se aplica un método de diagnóstico que se basa en indicadores que evalúan el potencial turístico del paisaje (Méndez-Méndez et al., 20r8), el cual es considerado como un instrumento básico para una adecuada planeación de la actividad turística en comunidades rurales. El método está dirigido a localidades que hayan manifestado un interés común por desarrollar la actividad turística o, en su caso, a localidades que, si bien ya la desarrollan, presentan profundos problemas relacionados con la falta de autenticidad y singularidad de los productos que ofertan al turista, lo que limita el potencial y la sustentabilidad del turismo (Tarroja, 2004; Meng y Uysal, 2008; Lane y Kastenholtz, 2015; Jerez y Serrano de la Cruz, 2016). Por esta razón, las mayores expectativas del estudio se tienen en aquellos lugares donde se presume una alta disponibilidad de recursos naturales y culturales, en donde es posible diseñar proyectos turísticos exitosos que respondan a los intereses de la comunidad y del turista.

El estudio presente se desarrolló en un área que es representativa de la diversidad biofísica y cultural de las principales ciudades del centro de México, como la Ciudad de México, Guadalajara, Puebla y Toluca, en donde existe una alta demanda por espacios naturales y rurales para la recreación y el esparcimiento de sus habitantes. Esta situación ha favorecido a que el turismo se desarrolle en una gran cantidad de localidades rurales que, a través de esta actividad, pretenden generar ingresos económicos complementarios a los que reciben de las actividades tradicionales y, de esta manera, contrarrestar las condiciones de pobreza y las complejas perspectivas de desarrollo en las que las comunidades subsisten.

La evaluación del potencial turístico del paisaje en estas zonas se realizó de acuerdo con un método integral que contempla la calidad intrínseca de los recursos geomorfológicos, biológicos y culturales de los paisajes identificados, así como de los atractivos turísticos que se encuentran en ellos. Para esto, se definieron unos indicadores objetivos y mensurables que pudieran describir, en cada caso, las características escénicas, ambientales e interpretativas que le dan valor de calidad a los recursos y atractivos turísticos del paisaje. La intención, finalmente, era que de dicho estudio se derivara la construcción de una propuesta útil para el desarrollo rural, específicamente, en el diseño e implementación de esquemas de planificación y gestión para el impulso del turismo en el área de estudio.

\section{Área de estudio}

La investigación se llevó a cabo en el municipio Isidro Fabela (Estado de México, $79,65 \mathrm{~km}^{2}$ ), ubicado en la vertiente oriental de la Sierra de Las Cruces, a $35 \mathrm{~km}$ de la Ciudad de México al noreste (figura I). El desnivel altitudinal, de I200 m (2400 a $3600 \mathrm{~m} \mathrm{s.} \mathrm{n.} \mathrm{m.),} \mathrm{favorece} \mathrm{a} \mathrm{la} \mathrm{diversidad} \mathrm{ambiental,} \mathrm{en} \mathrm{la} \mathrm{que} \mathrm{se} \mathrm{incluyen,} \mathrm{de} \mathrm{acuerdo} \mathrm{con} \mathrm{el} \mathrm{Instituto}$ Nacional de Estadística y Geografía (Inegi, 1983), bosques de encino (Quercus spp.) de la base de la sierra, bosques mixtos (encinos y coníferas), bosques de coníferas (pinos y abetos) y pinares de alta montaña (Pinus hartwegii). El patrón de uso del suelo en este municipio es, predominantemente, de superficie forestal (71,5\% del total), seguida por cultivos agrícolas (I8,7\%), asentamientos humanos (6,5\%) y áreas con vegetación secundaria (2,8\%) (GutiérrezLópez, 2018). 


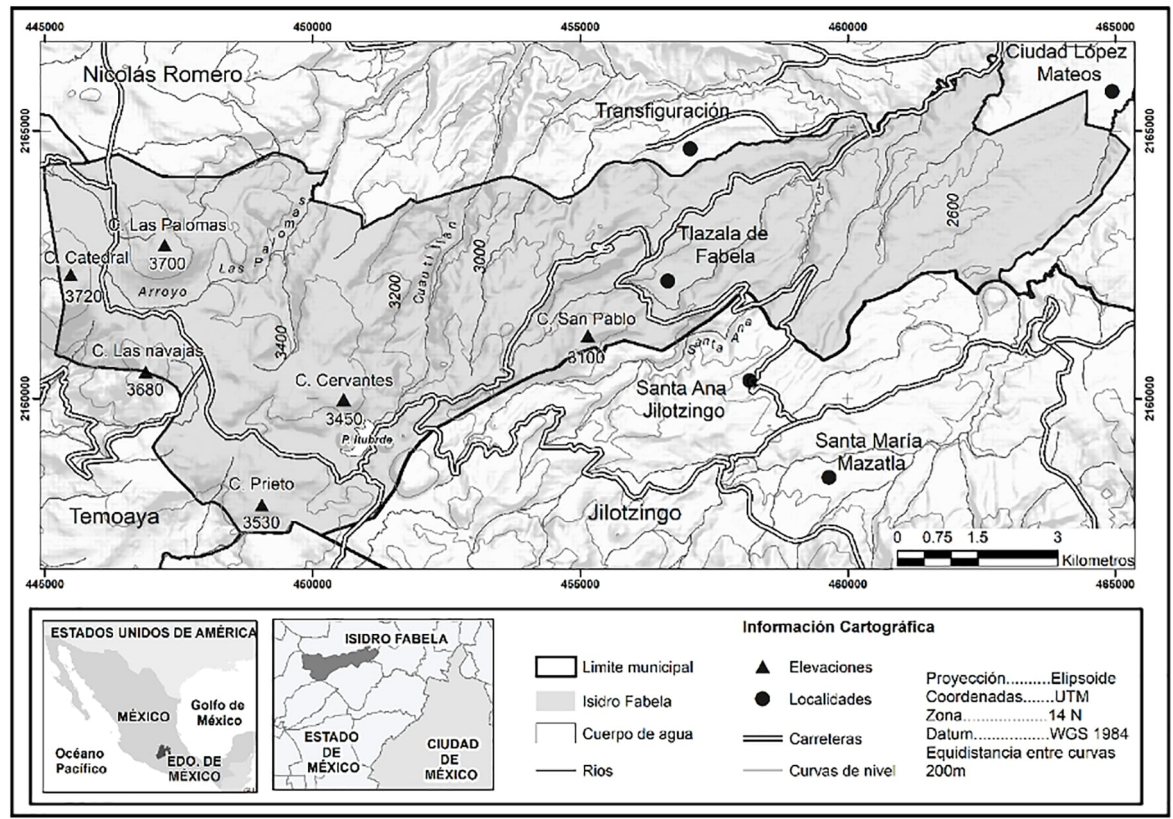

FIGURA 1.

Ubicación del municipio Isidro Fabela (Estado de México)

Fuente: Elaboración Propia EN SIG ArcMap 10.3

De acuerdo con el censo poblacional (Inegi, 2010), el municipio tiene 10.308 habitantes que se dedican, principalmente, a la agricultura, la ganadería y la acuicultura basada en la cría y venta de la trucha arcoíris (Oncorbynchus mykiss). La agricultura, cuya producción se destina al autoconsumo y una mínima parte al mercado local y externo, se desarrolla, por lo general, en las laderas tendidas de los barrancos del piedemonte serrano; el trigo, la avena y el maíz son los principales productos cultivados. En las últimas décadas, la actividad agraria ha tendido a disminuir debido a los bajos precios de compra y al incremento denle el costo de los agroquímicos (Escalante, 2017). Esta situación se ve reflejada en la caída del porcentaje de población que se ocupa en el sector agrícola que, para el año 2015 , representó solo el $15,24 \%$, mientras que el sector servicios se posicionó con el 62,94\% y la industria con el 21,72\% (Instituto de Información e Investigación Geográfica, Estadística y Catastral del Estado de México [Igecem], 2016).

De igual forma a como ocurre en otras comunidades rurales próximas a la capital del país, caracterizadas por la marginación, el rezago social y la sobreexplotación de los recursos naturales, la comunidad de Isidro Fabela mostró un creciente interés, desde la década de los ochentas, por desarrollar el turismo que, a la fecha, se ha mantenido como una actividad complementaria a los usos tradicionales del territorio, los cuales están en declive. En este estudio se abordan los casos de algunos atractivos turísticos que operan regularmente: Rancho Encantado, Valle de la Luna, Los Organillos, Presa Iturbide, Centro Recreativo Monarca, Rincón Bonito y Truchas El Ocotal, los cuales operan bajo el esquema de centros ecoturísticos y ofrecen diversas actividades recreativas (ciclismo, senderismo, tirolesa, recorrido a caballo, talleres, etc.) y servicios a los visitantes. En cuanto a la infraestructura en el interior de las instalaciones, los atractivos turísticos del municipio cuentan, por lo general, con estacionamiento, instalación eléctrica, área de juegos infantiles, granja y criadero de truchas.

Aunque la proximidad a la Ciudad de México supone para el área la posibilidad de un importante mercado, la mayoría de los atractivos turísticos operan con diversas dificultades, por ejemplo, la escasa calidad del producto que ofrecen, impactos ambientales que comprometen la sostenibilidad del producto, baja capacidad para la difusión de su oferta recreativa, gastronómica y paisajística, etc. Estas problemáticas muestran la ausencia de una planeación integral (biofísica, económica y social) y sugiere la necesidad de realizar una valoración del estado actual del 
potencial real que tienen estos atractivos, la cual sea una base para diseñar una adecuada planificación de la actividad turística.

\section{Metodología}

\section{Identificación, caracterización y cartografía de los paisajes y los atractivos turísticos}

En el presente estudio, se elaboró un mapa con los tipos de paisaje, el cual contiene las unidades de paisaje que forman su patrón paisajístico. Estos niveles taxonómicos son homogéneos en relación con los componentes y procesos físicos, biológicos y culturales del paisaje, los cuales lo definen y lo diferencian de los otros (Muñoz, 1998; García-Romero y Muñoz, 2002; Bertrand y Bertrand, 2006; Mateo, 2008).

Tipo de paisaje. El paisaje de dimensión media (decenas a cientos de $\mathrm{km}^{2}$ ) es el resultado de la combinación de una unidad superior de relieve y de una variante del clima regional. De estos dos componentes mayores dependen los recursos orográficos, altitudinales, de orientación, litológicos y climáticos -en particular, térmicos y pluviométricos-. Estos recursos, en conjunto, determinan las características del patrón paisajístico, el cual está compuesto por las unidades de paisaje contenidas en su interior.

Unidad de paisaje. El paisaje de pequeña dimensión (decenas a cientos de $\mathrm{m}^{2}$ ) está definido por la combinación de los componentes abióticos - hidrología, relieve y suelo- que influyen sobre otros componentes de carácter biótico y cultural -vegetación y uso del suelo-, los cuales son altamente inestables, dependientes y dinámicos. Las unidades de paisaje contenidas en el interior de un tipo de paisaje comparten el mismo ambiente climático y geomorfológico.

El mapa de unidades de paisaje (área mínima cartografiable de i ha) se elaboró en el SIG ArcMap i0.3, a partir de la integración tipológica y cartográfica de los mapas de formas del relieve con las formas de vegetación y el uso del suelo. Las formas del relieve fueron definidas como entidades homogéneas en el tipo de relieve, la litología asociada al origen, la edad del relieve y la geometría de las laderas (Méndez-Méndez et al., 20I8). La vegetación y el uso del suelo se interpretaron sobre ortofotos digitales (Inegi, 1999), por medio de técnicas directas, asociativas y deductivas para diferenciar los distintos elementos físicos de origen natural y cultural que cubren la superficie del terreno (Chuvieco, 2002). La integración de ambos parámetros se hizo mediante superposición de capas y reinterpretación, al atender al criterio de reagrupación de paisajes con un mismo significado funcional, en términos de la naturalidad (paisajes con mayor o menor carga del contenido natural y cultural), la estabilidad ambiental (asociada a los atributos geomorfológicos y de la vegetación) y el tipo, intensidad y permanencia de los usos del suelo (García-Romero y Muñoz, 2002).

Recientes investigaciones sobre el área de estudio señalan la existencia de más de cien atractivos turísticos en el municipio (Villegas-Martínez, 2016; Villegas-Martínez et al., 2019), de los cuales, en este estudio, se seleccionaron veintidós, los cuales fueron reconocidos durante una serie de visitas programadas al área de estudio, según las recomendaciones de varios informantes clave del municipio. Los atractivos seleccionados se destacan, además, porque se ubican a lo largo de los principales accesos carreteros y cuentan con señalamientos que facilitan la llegada de los visitantes, incluso, en temporada de baja afuencia. Se consideraron los atractivos turísticos identificados como producto directo de la investigación, así como los atractivos turísticos ya establecidos. En cada caso, los indicadores de calidad propuestos fueron documentados en campo, siguiendo el método Delphi (Gordon, 1994; 
Mateos, 2016). Los resultados obtenidos se cartografiaron y se introdujeron en la base de datos asociada para su posterior análisis.

\section{El potencial turístico: integración de la calidad de los tipos de paisaje y de sus atractivos turísticos}

La calidad turística de los tipos de paisaje se evaluó mediante el diseño y la aplicación de indicadores que hacen referencia a tres criterios de valoración: el escénico, el ambiental y el interpretativo.

Escénico: evalúa la cantidad y calidad visual de los componentes que definen el patrón del paisaje local. Este criterio valora el contraste visual que se obtiene entre las formas, los colores, la perspectiva resultante y la presencia de componentes excepcionales de alto valor. En este sentido, se considera tanto el valor escénico del sitio como su amplitud panorámica, es decir, la posibilidad de obtener vistas de larga distancia sin obstáculos, desde sitios puntuales o sobre trayectos longitudinales.

Ambiental: evalúa el grado de conservación de los factores bióticos y abióticos naturales, así como su relación con la estabilidad geomorfológica del paisaje. Asimismo, valora la productividad, sostenibilidad, accesibilidad e instalaciones asociadas a los componentes culturales. Lo anterior plantea la importancia que tiene el patrimonio material (por ejemplo, la infraestructura) e intangible (cultural e histórico-espiritual) que está asociado con el territorio, así como la originalidad e importancia de la relación entre los componentes del paisaje y el peso de algunos de ellos para la administración o protección de los demás.

Interpretativo: evalúa las características didácticas y de ejemplaridad del lugar, las cuales son representativas de la singularidad de los componentes del paisaje y su importancia para que, de ellas, se deriven las acciones educativas o el interés en los estudios académicos. Las características importantes, a este respecto, son el alcance relacionado tanto con la calidad de los componentes locales como con la configuración general del paisaje.

Para evaluar la calidad turística de los tipos de paisaje se contemplaron seis indicadores. Para el valor escénico, se utilizaron tres índices: el índice de diversidad de Simpson (1949), para medir la probabilidad de que dos unidades de paisaje seleccionadas al azar sean de la misma categoría dentro del tipo de paisaje; el índice de Shannon-Wiener, para predecir la categoría a la que pertenece la unidad de paisaje, y el índice de Jaccard, que mide el porcentaje de semejanza en la composición de los tipos de paisaje. Para el valor ambiental, por su parte, se utilizaron dos índices: el índice de forma, que mide la relación entre el área y el perímetro para el conjunto de unidades dentro del tipo de paisaje, y el número de parches totales dentro del tipo de paisajes. Para el valor interpretativo, se utilizó el índice de singularidad y/o representatividad del patrón paisajístico, el cual mide dentro del tipo de paisaje la superficie que presenta usos del suelo activos, relieve estable y vegetación conservada.

Para conocer la calidad de los atractivos e integrarla como valor añadido al potencial turístico de los tipos de paisaje, se utilizaron veintinueve indicadores. Estos valoran cada uno de los componentes básicos del paisaje (biológico, geomorfológico y cultural) con respecto a los tres criterios de valoración (tabla I). Es importante señalar que los indicadores propuestos fueron identificados con el apoyo de informantes locales clave, quienes están involucrados en el turismo. 
TABLA 1.

INDICADORES PARA EVALUAR LA CALIDAD DEL PAISAJE DE LOS ATRACTIVOS TURísticos

\begin{tabular}{|c|c|c|}
\hline Criterio escénico & Criterio ambiental & Criterio interpretativo \\
\hline \multicolumn{3}{|c|}{ Indicadores del componente biológico del paisaje } \\
\hline \multirow{3}{*}{ Tipo de vegetación } & Estado de conservación de la vegetación & Representatividad \\
\hline & Cercanía a áreas naturales protegidas & \multirow{2}{*}{ Singularidad } \\
\hline & Servicios ambientales & \\
\hline \multicolumn{3}{|c|}{ Indicadores del componente geomorfológico del paisaje } \\
\hline Calidad escénica intrínseca & \multirow{2}{*}{ Integridad } & \multirow{2}{*}{ Representatividad } \\
\hline Desnivel topográfico & & \\
\hline Presencia de cuerpos de agua & \multirow{2}{*}{ Asociado a la conservación del ecosistema } & \multirow{4}{*}{ Singularidad } \\
\hline Amplitud panorámica & & \\
\hline Variabilidad paisajística & \multirow{2}{*}{ Asociado a los rasgos culturales de valor paisajístico } & \\
\hline Longitud del tramo con panorámicas & & \\
\hline \multicolumn{3}{|c|}{ Indicadores del componente cultural del paisaje } \\
\hline \multirow{3}{*}{$\begin{array}{l}\text { Calidad escénica de la superficie } \\
\text { cultivada }\end{array}$} & Trascendencia religiosa o mística & \multirow{3}{*}{ Representatividad } \\
\hline & Trascendencia histórica & \\
\hline & Trascendencia artística o cultural & \\
\hline \multirow{5}{*}{$\begin{array}{l}\text { Calidad escénica del asentamiento } \\
\text { humano }\end{array}$} & Productos económicos de interés turístico & \multirow{5}{*}{ Singularidad } \\
\hline & Nivel de alcance del atractivo & \\
\hline & Equipamiento & \\
\hline & Tipo de acceso & \\
\hline & Distancia al sitio & \\
\hline
\end{tabular}

Fuente: elaboración propia

Cada uno de los indicadores utilizados fue calificado con una escala ordinal de cinco valores $(0,0,25,0,5,0,75$ y I), en la que i indica la calidad más alta que se puede tener en un sitio. A partir de estas calificaciones, se determinó la calidad de cada atractivo turístico (CAT), con el resultado de la suma de las puntuaciones obtenidas por los distintos indicadores, referentes a sus componentes biológico, geomorfológico y cultural. Para evitar la sobre o subestimación de los componentes, se utilizaron los criterios de ponderación propuestos por Méndez-Méndez et al. (2018).

Dado que en este estudio se utilizaron veintinueve indicadores, los atractivos turísticos se clasificaron en función de su valor CAT, en relación con su máximo valor posible (CAT máx $=29$ ), cuando todos los indicadores tienen la puntuación más alta (I). Así, se definieron tres categorías de calidad: baja (inferior a 9,67), media (entre 9,67 y 19,36) y alta (entre 19,37 y 29).

El potencial turístico de los paisajes del municipio Isidro Fabela se difiere a partir de la calidad expresada por cada uno de los indicadores; de esta forma, la calidad de los atractivos turísticos se integró a la calidad de los tipos de paisaje, este como valor añadido que aumenta su potencial turístico. Al ser los indicadores dimensionalmente independientes entre sí, se interpretaron por separado, de tal manera que la calidad de los tipos de paisajes y de los atractivos turísticos no corresponde a la suma simple de dichos valores, sino al resultado de su interpretación cualitativa y pragmática. Con base en el significado y los umbrales propios, cada indicador se asoció a una de las tres categorías establecidas (CAT= baja, media y alta), lo que permitió homogenizar los resultados y facilitar su interpretación. 


\section{Análisis de resultados}

\section{Clasificación y valoración de la calidad turística de los tipos de paisaje}

Los paisajes del municipio Isidro Fabela se organizan en dos tipos de paisaje y cincuenta unidades de paisaje. Los primeros corresponden a las laderas y cumbres con bosque de coníferas, de uso forestal-turístico, y al piedemonte piroclástico con bosque mixto, de uso agrícola y asentamientos dispersos. Ambos tipos de paisaje son representativos de la diversidad paisajística que caracteriza a los dos principales ámbitos geomorfológicos y bioclimáticos de la Sierra de Monte Alto (figura I).

Laderas y cumbres con bosque de coniferas, de uso forestal-turístico (LC). Unidad conformada por altas cumbres y laderas de montaña (>2800 m s. n. m.), donde los climas cambiantes del Cuaternario provocaron extensos valles y cabeceras, encajados sobre lavas fracturadas del Terciario, lo que hace que sean frecuentes los escarpes erosivos, afectados por procesos de gravedad y fluviales. El desnivel topográfico supera los $500 \mathrm{~m}$ en la mayor parte del área, lo que favorece las precipitaciones orográficas (>1000 mm/año) y una drástica transición de los climas templados a los frescos en las cumbres. La vegetación potencial corresponde al piso bioclimático de los bosques de coníferas, los cuales cubren una superficie equivalente al $44,8 \%$ de la entidad. Podría decirse que la topografía agreste limita el desarrollo agropecuario, razón por la cual el patrón paisajístico es poco diverso, aunque, en las últimas décadas, los recursos forestales y orográficos han favorecido el desarrollo del turismo.

Piedemonte piroclástico con bosque mixto, de uso agricola y asentamientos dispersos (PP). Esta unidad abarca el sector alto del piedemonte de la sierra, caracterizado por una compleja serie de capas vulcano-detríticas del Plio-Cuaternario. Los materiales originales de la zona resultaron fracturados por la actividad tectónica y la erosionados causada por las corrientes fluviales, lo que tuvo como resultado la conformación de extensos valles asimétricos, profundamente encajados $(>200 \mathrm{~m})$. El clima templado de las cumbres permite el desarrollo de una vegetación potencial que corresponde al piso de los bosques mixtos de coníferas y frondosas. El patrón paisajístico se caracteriza por tener áreas forestales que se distribuyen y están confinadas en las laderas abruptas de los valles asimétricos, en tanto que, en las laderas tendidas, terrazas aluviales y valles de fondo plano, el patrón está dominado por extensas áreas de cultivo con asentamientos humanos dispersos.

La calidad de los tipos de paisaje se obtiene de la valoración del conjunto de unidades que definen su patrón paisajístico. En cuanto a su valor escénico, los valores obtenidos muestran una situación similar en ambos tipos de paisaje. Así, el índice de Simpson arrojó valores bajos (<I), los cuales reflejan una escasa posibilidad de dominancia de alguna de las categorías y, por lo tanto, una mayor diversidad paisajística, ligeramente superior en las laderas y cumbres. El mismo comportamiento se observó con el índice de Jaccard, en el que los valores bajos (<I) reflejan una alta disimilitud entre ambos tipos de paisaje. En cambio, ambos tipos de paisaje obtuvieron valores bajos para el índice de Shannon-Wiener (por debajo de $\mathrm{H}^{\prime}=2,0$ ), lo que refleja una baja diversidad específica, ligeramente superior en el piedemonte piroclástico. 
TABLA 2.

Evaluación de la CAlidad de los tipos de PAISAJE

\begin{tabular}{|c|c|c|c|c|c|c|}
\hline \multirow[t]{2}{*}{ Tipos de paisaje } & \multicolumn{3}{|c|}{ Valor escénico } & \multicolumn{2}{|c|}{ Valor ambiental } & \multirow{2}{*}{ 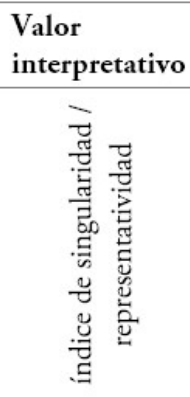 } \\
\hline & 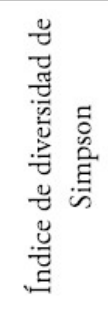 & 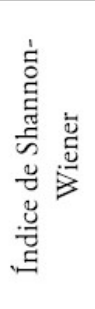 & 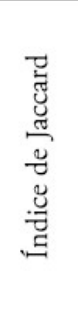 & 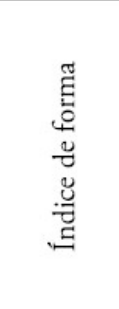 & 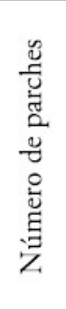 & \\
\hline $\begin{array}{l}\text { Tipo 1. Laderas y cumbres con bosque de coníferas, } \\
\text { de uso forestal-turístico (LC) }\end{array}$ & 0,88 & 2,49 & 0,14 & 262,73 & 83 & 0,99 \\
\hline $\begin{array}{l}\text { Tipo 2. Piedemonte piroclástico con bosque mixto, } \\
\text { de uso agrícola y asentamientos dispersos (PP) }\end{array}$ & 0,94 & 3,22 & 0,14 & 475,41 & 120 & 1 \\
\hline
\end{tabular}

Fuente: elaboración propia

El valor ambiental que tienen los tipos de paisaje se definió a partir de dos indicadores de la fragmentación espacial, considerada aquí como un factor ambientalmente adverso y que refleja el deterioro, la falta de estabilidad y la escasa resiliencia de los atributos naturales del paisaje (tabla 2). Tanto el índice de forma como el número de parches son menores en las laderas y cumbres con respecto al piedemonte piroclástico, el cual se caracteriza por tener una mayor ocupación antropogénica y complejidad del patrón del paisaje, en torno al pueblo de Tlazala.

Finalmente, los valores obtenidos para el índice de singularidad/representatividad, en ambos tipos de paisaje, demuestran la existencia en el área de destacados recursos de carácter interpretativo, de gran interés académico y educativo. En conjunto, estos datos muestran una calidad relativamente homogénea entre las laderas y cumbres y el piedemonte piroclástico del municipio, aunque el patrón de paisaje del primero se destaca por tener valores que son ligeramente más favorables en la mayoría de los indicadores utilizados.

\section{Inventario y valoración de la calidad de los atractivos turísticos}

La información obtenida de los informantes clave del municipio permitió establecer un inventario de veintidós atractivos turísticos, distribuidos de la siguiente manera: doce en las laderas y cumbres y diez en el piedemonte piroclástico. Cabe señalar la existencia de otros atractivos con potencial turístico o atractivos con desarrollo turístico, los cuales se diferencian por la existencia o no de infraestructura e información al turista, así como por el tipo de actividades que se ofrecen. Para facilitar el estudio, los atractivos se dividieron en las siguientes cinco categorías.

Atractivos naturales sin equipamiento. Son espacios amplios que presentan un alto potencial para desarrollar actividades recreativas, en contacto con la naturaleza, pero que carecen de accesos, estacionamientos, instalaciones para el turismo y venta de alimentos, entre otros equipamientos y servicios. Estos espacios son conocidos y utilizados por la población local, en tanto que el turismo foráneo es ocasional.

Atractivos culturales. Espacios con temática histórico-cultural, en donde se pueden reconocer diversos aspectos de la apropiación histórica por parte de la población local. Pueden o no contar con equipamientos y servicios básicos 
para el desarrollo de la actividad turística, la cual se caracteriza por la llegada ocasional de visitantes y por una derrama económica poco significativa.

Trayectos y miradores panorámicos. Comprende los atractivos turísticos de tipo lineal -principalmente, segmentos de carretera- y de tipo puntual, los cuales ofrecen vistas panorámicas de calidad.

Atractivos naturales con equipamiento. Son establecimientos en operación, con un área delimitada en el territorio y que son reconocidos por ofertar diversas actividades turísticas. Aunque el nivel de desarrollo es variado entre los distintos atractivos, existen claras semejanzas en cuanto al tipo y calidad de los equipamientos y servicios que ofrecen, principalmente, el acceso asfaltado y el espacio de estacionamiento, la oferta de diversas actividades de recreación y de alimentos en tiendas o comedores, además de restaurantes para el consumo de trucha.

Venta de alimentos. Son aquellos establecimientos dedicados a la cría y venta de trucha.

La evaluación de la calidad paisajística de los atractivos turísticos se obtuvo a partir de veintinueve indicadores: nueve para calificar el valor escénico; catorce, el valor ambiental, y seis, el valor interpretativo de los componentes del paisaje (biológico, geomorfológico y cultural) (tabla z).

El conjunto de los veintidós atractivos turísticos identificados en Isidro Fabela obtuvo una puntuación baja $(C A T=8,58)$, debido a la ausencia de atractivos de alta calidad y del predominio de los atractivos de baja calidad $(C A T=2,5$ a 9,5) sobre los de calidad media $(C A T=9,75$ a 13,5). Los indicadores ambientales que más contribuyeron al $C A T$ son los relacionados con el relieve $\left(V_{g}=3,55\right)$, seguidos por los indicadores culturales $\left(V_{c}=2,60\right)$ y de la vegetación $(V b=2,47$ ) (tabla 3 ). El centro recreativo Valle de la Luna fue el atractivo que obtuvo la mayor puntuación $(C A T=13,5)$, dado que es el sitio más conocido y visitado del municipio. Este se ubica en una extensa vega que se forma ladera arriba del embalse del centro recreativo Presa Iturbide; las laderas lávicas y domos cubiertos por bosque de coníferas que la circundan conforman un paisaje que cautiva a sus visitantes.

El atractivo turístico con la segunda mayor puntuación fue el sendero del río Cuautitlán $(C A T=12,5)$. En este trayecto, el visitante puede apreciar la estructura interna de un barranco notablemente incidido por la red fluvial, con un modelado característico de los procesos fluviales y de gravedad que afectan las capas de piroclastos y lavas que afloran en las laderas verticales del barranco. También se pueden apreciar escalones rocosos y cascadas de ambiente umbroso y húmedo, en el que destaca la compleja estructura y la diversidad biológica de un bosque mixto en ambiente de galería.

Finalmente, el centro recreativo Los Organillos obtuvo la tercera posición (CAT= II,8), ubicado en el interior de un barranco secundario próximo al centro recreativo Valle de la Luna. Este recibe pocos visitantes, a pesar de contar con una mejor planeación y organización de las actividades recreativas, lo que se refleja en mejores condiciones del ambiente biológico y geomorfológico. 
TABLA 3

INVENTARIO Y VALORACIÓN DE LA CALIDAD DE LOS ATRACTIVOS TURÍSTICOS

\begin{tabular}{|c|c|c|c|c|c|c|c|c|}
\hline Núm. & Atractivo turístico & $\begin{array}{l}\text { Tipo de } \\
\text { paisaje }\end{array}$ & Unidad de paisaje & $\mathrm{Vb}$ & $\mathrm{Vg}$ & Vc & $C A T$ & Rango \\
\hline 1 & Centro recreativo Valle de la Luna & LC & Montaña lávica con bosque de pino & 4,75 & 5,75 & 3 & 13,50 & Medio \\
\hline 2 & Sendero del río Cuautitlán & LC & Barranco con bosque de coníferas & 5,50 & 6 & 1 & 12,50 & Medio \\
\hline 3 & Centro recreativo Los Organillos & LC & Montaña lávica con bosque de pino & 4,75 & 4 & 3 & 11,80 & Medio \\
\hline 4 & Área de venta de pinos & LC & Montaña lávica con cultivo de temporal & 3,25 & 5,50 & 2,75 & 11,50 & Medio \\
\hline 5 & Centro recreativo Presa Iturbide & LC & Barranco con bosque de pino & 3,75 & 4,25 & 3 & 11 & Medio \\
\hline 6 & Mirador del Valle las Palomas & LC & Montaña lávica con bosque de pino & 3,75 & 5 & 1,75 & 10,50 & Medio \\
\hline 7 & Temazcal el quinto sol & PP & $\begin{array}{l}\text { Asentamientos humanos dispersos con cultivos de } \\
\text { temporal en laderas complejas }\end{array}$ & 1 & 4 & 5 & 10 & Medio \\
\hline 8 & Presa Iturbide - Tlazala de Fabela & LC & Montaña lávica con bosque de coníferas & 3,25 & 5 & 1,50 & 9,75 & Medio \\
\hline 9 & Área verde Llano Tecuani & LC & Montaña lávica con pastizal secundario & 2,25 & 5,25 & 2 & 9,50 & Bajo \\
\hline 10 & Aparcelado del valle El Mongo & LC & Montaña lávica con bosque de coníferas y latifoliadas & 1,75 & 4,25 & 3,25 & 9,25 & Bajo \\
\hline 11 & $\begin{array}{l}\text { Centro recreativo Rancho } \\
\text { Encantado }\end{array}$ & PP & Barrancos con bosque de encino & 3,25 & 2,50 & 3,25 & 9 & Bajo \\
\hline 12 & Área verde Llano Navajas & LC & Montaña lávica con pastizal secundario & 2,25 & 4,50 & 2 & 8,75 & Bajo \\
\hline 13 & $\begin{array}{l}\text { Trayecto panorámico del valle El } \\
\text { Mongo }\end{array}$ & LC & Montaña lávica con cultivo de temporal & 2,75 & 4 & 1,50 & 8,25 & Bajo \\
\hline 14 & Zócalo de Tlazala de Fabela & PP & $\begin{array}{l}\text { Asentamientos humanos dispersos con cultivos de } \\
\text { temporal en laderas complejas }\end{array}$ & 0 & 2,75 & 5,25 & 8 & Bajo \\
\hline 15 & Venta de truchas El Ocotal & PP & $\begin{array}{l}\text { Asentamientos humanos dispersos con cultivos de } \\
\text { temporal en terraza aluvial }\end{array}$ & 2 & 3,50 & 2,50 & 8 & Bajo \\
\hline 16 & Centro recreativo Rincón Bonito & LC & Montaña lávica con bosque de coníferas y latifoliadas & 2 & 3,50 & 2,50 & 7 & Bajo \\
\hline 17 & Centro recreativo Monarca & PP & $\begin{array}{l}\text { Asentamientos humanos dispersos con cultivos de } \\
\text { temporal en laderas tendidas complejas }\end{array}$ & 2,50 & 0,75 & 3 & 6,25 & Bajo \\
\hline 18 & Venta de truchas El Huerto & $\mathrm{PP}$ & $\begin{array}{l}\text { Asentamientos humanos dispersos con cultivos de } \\
\text { temporal en laderas complejas }\end{array}$ & 2,25 & 1,25 & 2,25 & 5,75 & Bajo \\
\hline 19 & Venta de truchas Flipper & $\mathrm{PP}$ & Ladera tendida con cultivos de temporal & 1,50 & 1,50 & 2,75 & 5,75 & Bajo \\
\hline 20 & Mirador de Tlazala de Fabela & PP & $\begin{array}{l}\text { Asentamientos humanos dispersos con cultivos de } \\
\text { temporal en laderas tendidas complejas }\end{array}$ & 0 & 3 & 2,25 & 5,25 & Bajo \\
\hline 21 & Balncario/hotel Pueblo Bonito & $\mathrm{PP}$ & Barranco con bosque de encino & 0 & 1,50 & 3,50 & 5 & Bajo \\
\hline 22 & Venta de truchas El Paraíso & PP & $\begin{array}{l}\text { Asentamientos humanos dispersos con cultivos de } \\
\text { temporal en laderas tendidas complejas }\end{array}$ & 2 & 0,25 & 0,25 & 2,50 & Bajo \\
\hline Promedio & & & & 2,47 & 3,55 & 2,60 & 8,58 & Bajo \\
\hline
\end{tabular}

Fuente: elaboración propia

$V b=$ Valor biológico

$V g=$ Valor geomorfológico

$V_{c}=$ Valor cultural

Cat $=$ Calidad del atractivo turístico

\section{Síntesis del potencial turístico del paisaje: integración de la calidad turística de los tipos de paisaje y de sus atractivos turísticos}

La figura 2 muestra la distribución de los atractivos turísticos en los diferentes tipos de paisaje. La valoración de la calidad del patrón de paisaje y de los atractivos turísticos contenidos en los dos tipos de paisaje reconocidos en el municipio se resuelve en un mayor potencial turístico, el cual está asociado a las laderas y cumbres con bosque de coníferas, de uso forestal-turístico (LC). El valor alcanzado por esta unidad fue comparativamente superior en la mayoría de los indicadores de calidad de paisaje, tanto escénica (índices de Simpson y Jaccard), ambiental (índice de forma y número de parches) y de singularidad/representatividad (tabla 2). Asimismo, es de destacar que de los veintidós atractivos turísticos que se han trabajado en el municipio, doce se encuentran en LC, incluidos siete de los ocho atractivos de mayor calidad (CAT= medio). Esto le atribuye a la unidad paisajística un alto interés turístico, favorecido, principalmente, por la calidad de sus recursos biológicos y geomorfológicos, así como por la 
presencia y relevancia, en el sector inferior de la montaña, de las obras de infraestructura (embalses artificiales) que impactan de manera positiva la calidad escénica y funcional del paisaje (tabla 3 ).

Por su parte, el piedemonte piroclástico con bosque mixto, de uso agrícola y asentamientos dispersos (PP) obtuvo una ligera desventaja en la valoración de su patrón paisajístico, el cual, no obstante, fue superior en uno de los indicadores de calidad escénica (índice de Shannon-Wiener), en tanto que los indicadores ambientales (índice de forma y número de parches), los cuales reflejan una mayor ocupación antropogénica y grado de perturbación, también se asocian a la diversidad cultural del paisaje en torno al pueblo de Tlazala (tabla 2). En el piedemonte se sitúan diez atractivos turísticos, incluidos los puntos de venta de trucha, los centros recreativos y los hoteles, de los cuales nueve fueron valorados como de baja calidad. Todos ellos están enclavados en ambientes rurales y ofertan servicios con base en los recursos biológicos y geomorfológicos del paisaje, lo que tiene evidentes impactos en el ambiente natural; cuentan, finalmente, con un equipamiento deficiente (tabla 3 ).

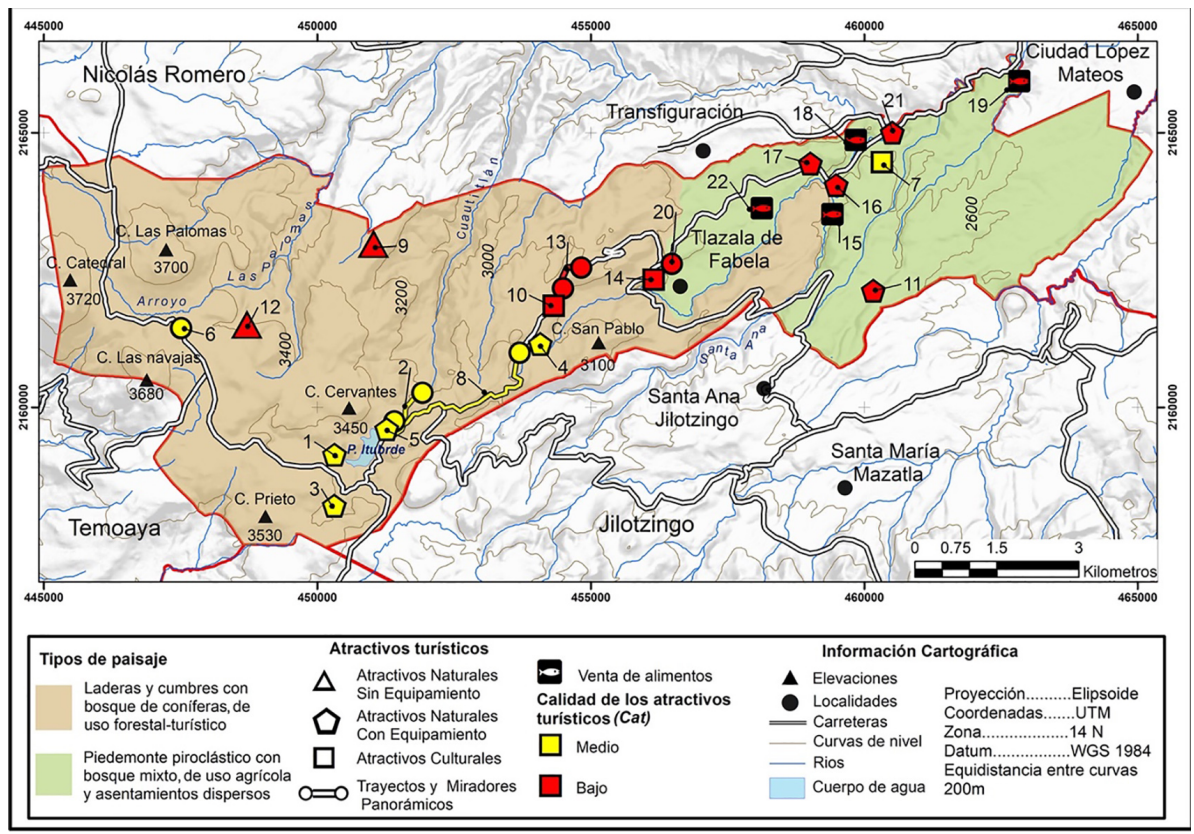

FIGURA 2.

Distribución de los atractivos turísticos EN los DifERENTES TiPos De PAisAJE

Nota: la numeración de los atractivos turísticos refleja su valor de calidad paisajística, siendo i el atractivo de mayor calidad y 22 el de menor calidad (véase la tabla 3).

Fuente: elaboración propia en SIG ArcMap 10.3 


\section{Discusión}

\section{Sobre el método de estudio: el uso de indicadores para el diagnóstico del potencial turístico del paisaje a distintos niveles de integración}

En esta investigación se propone un método para la identificación de los paisajes y atractivos turísticos de distinta calidad para, a partir de la información que brindan, fundamentar el desarrollo integral de la actividad turística, lo que trae beneficios a un sector más amplio de los territorios y las comunidades involucradas. Con ello, se fortalecen las actuales tendencias científicas e institucionales que buscan promover el paisaje como elemento de planificación y gestión (Council of Europe, 2000; OECD, 2003; Barbini, 2005; Mata, 20II). Estos análisis son particularmente útiles en el ordenamiento del uso del suelo, la protección del patrimonio natural y cultural y la participación pública (Gómez-Zotano et al., 20I8).

El método utilizado tiene un carácter eminentemente sistémico, a partir del punto de vista del análisis espacial, lo que permite presentar una visión multiescalar sólida que es heredera de los procedimientos clásicos de estudio del paisaje (Bolòs, 1992; Muñoz, 1998) y que se alinea con planteamientos de carácter geográfico (Vallés et al., 20I2). La clasificación del paisaje utilizada considera dos niveles (tipos de paisaje y unidades de paisaje) que aseguran la diferenciación de las relaciones espaciales y funcionales entre los componentes del ambiente (geomorfológico, biológico y cultural), los cuales ocurren tanto en los paisajes de un mismo nivel como entre paisajes de distinto nivel, tal y como dejan de manifiesto otros trabajos previos (García-Romero y Muñoz, 2002; Arias García et al., 2017; Gómez-Zotano et al., 2018). La integración de niveles tuvo como objetivo que la calidad de los atractivos turísticos se complete con la calidad de los tipos paisajísticos y que el resultado fungiera como valor añadido, el cual aumenta el potencial turístico del paisaje, por ejemplo, en lugares que de otra forma no podrían destacar para el turismo.

Se le concede particular importancia a los elementos estructurantes o diferenciadores del paisaje (Muñoz, 1998; García-Romero y Muñoz, 2002). En efecto, en el análisis y la síntesis del paisaje y, posteriormente, en la caracterización de las unidades, el método considera todos aquellos elementos con significado real en el paisaje, es decir, aquellos que contribuyen de manera significativa a la constitución de este (Mikulec y Antoušková, 20II). En particular, se atiende a las actuales tendencias que añaden al valor estético del paisaje (Zuluaga, 2006; Knudsen et al., 20I3; Kalivoda et al., 20I4; Nogué et al., 2019) otros valores, tales como el ecológico, educativo, interpretativo, histórico, etc., todos los cuales apuestan por la autenticidad y singularidad de los lugares (Torres, 2003; Tarroja, 2004; Meng y Uysal, 2008; Picazo, 20I2; Lane y Kastenholtz, 2015; Cebrián y García, 2016; Jerez y Serrano de la Cruz, 2016).

Para el cumplimiento de lo anterior, se diseñaron y aplicaron diversos indicadores de calidad, mensurables y objetivos que hacen referencia a los distintos componentes (biológico, geomorfológico y cultural), los cuales proporcionan una visión integrada del conjunto de recursos disponibles en el paisaje (Cawley y Gilmor, 2008). Esto ayudó a construir una evaluación altamente explicativa, sistemática e integral, con un mayor margen para objetivos prácticos de uso (Serrano, 2008; Nogué et al., 2009; Mearns, 2012). Además, se consideraron tres criterios de valoración de la calidad del paisaje (escénico, ambiental e interpretativo) que fueron claves para el desarrollo 
de una propuesta metodológica más adecuada, que promueva el turismo rural, sostenible e integrado (Butler, 1999; Kenafsey, 200I; Salinas y La O, 2006; Cawley y Gillmor, 2008; Prat Forga y Cànoves Valiente, 20r4) y con la posibilidad de ser aplicada en otras áreas rurales diferentes e incluso contrastantes.

\section{Sobre el potencial turístico del paisaje en Isidro Fabela}

Los resultados obtenidos permiten confirmar que el paisaje es un recurso que integra las condiciones naturales y culturales del territorio, algunas de las cuales pueden ser consideradas como elemento para el desarrollo del turismo (Escriche, 1999; Zuluaga, 2006; Dosso, 20II; Picazo, 20I2; Carneiro et al., 20I5; Cebrián y García, 2016). En ese sentido, los paisajes del municipio Isidro Fabela ofrecen condiciones adecuadas para el desarrollo turístico, espacio en el que se observan dos escenarios claramente diferenciados: las laderas y cumbres (LC) se asocian de manera consistente con dos modalidades del denominado turismo alternativo (ecoturismo y turismo de aventura), mientras que, sin ser excluyente de dichas modalidades, las áreas del piedemonte piroclástico (PP) se vinculan a una tercera variante, que es el turismo rural.

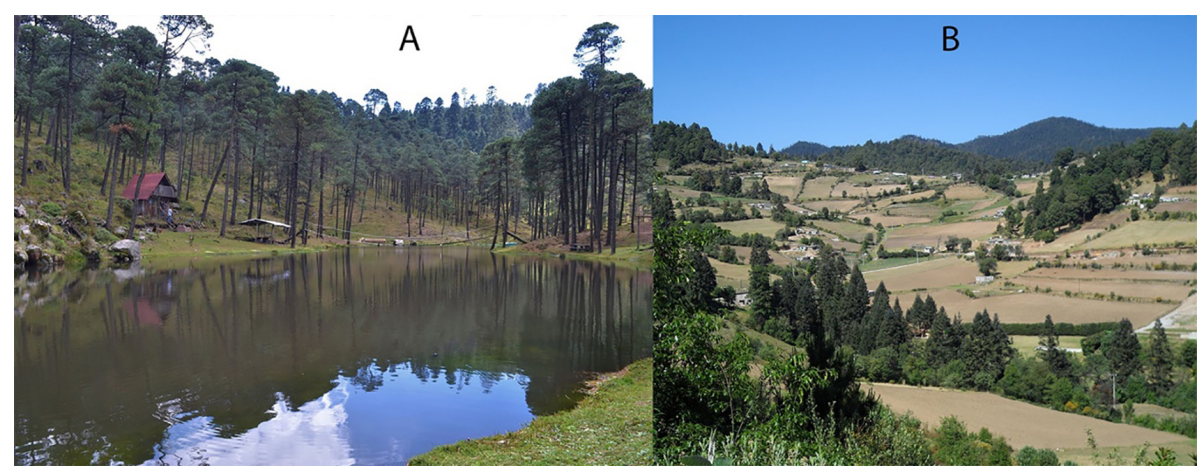

FIGURA 3.

Tipos DE PAISAJE

Nota: se observan dos paisajes característicos del paisaje de laderas cumbres. En (A) aparece la vista del embalse y de las laderas dómicas, cubiertas por bosques de pino, en el centro recreativo Los Organillos $(C A T=I I, 8)$. En (B) se observa la vista en un punto del trayecto panorámico

del Valle El Mongo, sobre la carretera Tlazala-Presa Iturbide, caracterizado por un parcelario con gran belleza escénica $(C A T=8,25)$. Fuente: Dalia Margarita Gutiérrez López (2015)\}

El patrón de paisaje de las LC se destacó por tener valores que son ligeramente más favorables en la mayoría de los indicadores utilizados. Así, el valor escénico mostró una clara disimilitud entre ambos tipos de paisaje (índice Jaccard), una baja dominancia que refleja una mayor diversidad paisajística -mayor en las LC- (índice de Simpson) y una baja diversidad específica (índice de Shannon-Wiener). El valor ambiental fue mayor en las LC (índice de forma y cantidad de parches), asociado a un patrón de fragmentación que sugiere un mejor grado de conservación del recurso forestal. El valor interpretativo (índice de representatividad/singularidad) mostró la existencia en el área de destacados recursos de gran interés académico y educativos En el interior de estos paisajes, se reconocieron veintidós atractivos turísticos: ocho de ellos fueron evaluados como de calidad media y se concentraban en el tipo de paisaje de las LC.

Particularmente, se identificaron diversos atractivos turísticos que pueden ser el objeto central de los proyectos detonadores de la actividad turística. Estos atractivos son, básicamente, aquellos de calidad media, con los cuales se puede impulsar el aprovechamiento de otros atractivos de menor calidad. Así, los paisajes y los atractivos de calidad turística le confieren al territorio de estudio un mayor potencial, por ejemplo, para el diseño de rutas turísticas 
que favorezcan el desarrollo integral de la actividad turística y una mayor capacidad para satisfacer, a partir del punto de vista de la demanda social, la micromovilidad de un extenso mercado (Lane y Kastenholz, 2015), el cual está concentrado en la Ciudad de México, así como proporcionar los beneficios de la actividad a un sector más amplio de la sociedad local.

De esta forma, el estudio presente aporta al éxito del turismo como actividad complementaria para afrontar la pobreza en el medio rural. Sin embargo, se requieren soluciones integrales que consideren la atención a otros problemas vinculados, por ejemplo, con la construcción de infraestructura básica -que usualmente debería ser provista por el sector público-(Lane y Kastenholz, 2015), el empoderamiento de los usos tradicionales del suelo, la solución de los conflictos relacionados con la propiedad de la tierra, la organización del comercio y la emigración de la población joven. En estos casos, el paisaje es entendido no solo como un elemento complementario, sino como un recurso y un apoyo básico para el turismo y para la creación del valor de los lugares (Aitchison et al., 200I; Nogué et al., 2009; Picazo, 2012; Knudsen et al., 2008, 2013; Wlodarczyk, 2009). El paisaje puede ser capaz de articular un turismo sostenible para el desarrollo rural, a través de la puesta en valor de sus características, del respeto a las comunidades rurales y de una planificación comedida que no transforme completamente la vocación productiva de estos espacios rurales y, por tanto, sus paisajes.

\section{Conclusiones}

Durante décadas, el turismo ha sido considerado como una actividad clave para la reestructuración económica de las áreas rurales en las que actividades como la agricultura y la cría de ganado han decaído, lo que perjudica el bienestar de la población. Sin embargo, muchos enfoques para el desarrollo de esta actividad han considerado el potencial turístico sin incluir una evaluación objetiva del paisaje y de sus atractivos. En este caso, la evaluación del paisaje en términos de calidad paisajística, ambiental y cultural se llevó a cabo mediante un enfoque pragmático que logra integrar, bajo un mismo esquema de diagnóstico, el conjunto de indicadores geosistémicos y de percepción que determinan la calidad del paisaje desde el punto de vista turístico. Asimismo, se consideró como criterio central de la ponderación el valor agregado, el cual se encuentra asociado a la frecuencia y calidad de los atractivos. Estos desempeñan un papel claramente definido, por ejemplo, productos que se pueden ofrecer de inmediato a los visitantes, lo que diversifica la gama de posibilidades del turismo alternativo (ecoturismo, turismo de aventura y turismo rural).

Se espera que este enfoque metodológico, basado en la combinación de criterios aplicados a dos niveles de análisis de los recursos turísticos (paisajísticos y de atractivos), pueda ser empleado en otras comunidades rurales de diferente naturaleza y que sirva como estudio base que apoye la adecuada planificación de los proyectos locales durante las etapas de diagnóstico. Además, el método es complementario a otros enfoques, como aquellos que impulsan la participación activa de las comunidades locales, elemento central para el desarrollo de proyectos de turismo responsable. También están aquellos estudios que se enfocan en la prevención del efecto ambiental adverso, el cual, con frecuencia, se ha observado en distintos proyectos de desarrollo turístico en su etapa operativa.

Finalmente, la evaluación del potencial turístico del paisaje, como ha sido presentado en esta investigación, puede contribuir al desarrollo económico de las áreas rurales, a través de la rigurosa valoración de los paisajes y sus atractivos de mayor autenticidad y singularidad. Con base en estos, deberá fundamentarse el desarrollo o reestructuración de los productos turísticos que ofertan al mercado. Es importante destacar que el reconocimiento de los recursos turísticos más significativos, de los cuales depende la actividad turística, será clave para potenciar el desarrollo de la actividad en el interior de las comunidades, lo que permite un reparto más equitativo de los 
beneficios obtenidos y disminuye el riesgo de pérdida de la identidad natural y de la herencia cultural que les pertenece.

\section{Referencias}

Aitchison, C., Macleod, N., y Shaw, S. (200I). Leisure and Tourism Landscapes: Social and Cultural Geographies. Routledge.

Arias García, J., Gómez Zotano, J., y Delgado Peña, J. J. (20I7). Classifying landscape in endorheic basins: a methodological approach for the implementation of the European Landscape Convention. European Journal of Geography, 8(2), 55-77. http://www.eurogeographyjournal.eu/articles/4.Classifying\%2olandscape\%20in\%2oendorheic\%2ob asins\% $20 \mathrm{a} \% 20$ methodological\%20approach\%20for\% 20 the\% 20 implementation $\% 20$ of $\% 20$ the $\% 20$ European $\% 20$ landscape\%2oconvention.pdf

Barbini, B. (2005). Viabilidad social para el desarrollo turístico en centros urbanos bonaerenses. Aportes $y$ transferencias, 9(2), I48-158. https://www.redalyc.org/pdf/276/27690209.pdf

Bertrand, C., y Bertrand, G. (2006). Geografía del medio ambiente: el sistema GTP: geosistema, territorio y paisaje. Universidad de Granada.

Bolòs, M. (1992). Manual de ciencia del paisaje. Masson.

Bucheli, M. S., Castillo, C. A., y Villarreal, J. L. (2009). Medir el patrimonio cultural: un desafío para la contabilidad. Cuadernos de Contabilidad, ro(26), 5I-65. http://www.scielo.org.co/pdf/cuco/vion26/vion26a03 .pdf

Butler, R. (1999). Sustainable Tourism: A State-of-the-Art Review. Tourism Geographies, I, 7-25. https://doi.org/ıO. 1080/14616689908721291

Carneiro, M. J., Lima, J., y Silva, A. L. (2015). Landscape and the rural tourism experience: identifying key elements, addressing potential, and implications for the future. Journal of Sustainable Tourism, 23 (8-9), 1217-1235. https://doi.org/10.1080/09669582.2015.1037

Cawley, M., y Gillmor, D. A. (2008). Integrated rural tourism: Concepts and Practice. Annals of Tourism Research, 35(2), 316-337. https://doi.org/10.1016/j.annals.2007.07.011

Cebrián, F., y García, C. (2016). Uso y gestión del paisaje para la actividad turística en el medio rural: aproximación teórica y empírica en el sureste de Castilla-La Mancha (Albacete). Boletín de la Asociación de Geógrafos Españoles, (72), 38I-407. https://doi.org/10.21138/bage.2345

Chuvieco, S. E. (2002). Teledetección Ambiental. La observación de la tierra desde el espacio. Ariel Ciencia.

Council Of Europe (2000). European Landscape Convention. European Treaty Series, N.o 176. Strasbourg.

Dosso, R. H. (20II). Turismo y paisaje: pluralidad conceptual, versatilidad interpretativa y responsabilidad interdisciplinar. Aportes y transferencias, 15(2), 15-54. http://nulan.mdp.edu.ar/id/eprint/1767

Escalante, S. (2017). Calidad del suelo y diversidad vegetal en milpas en Tlazala, Estado de México: percepción de diferentes sistemas de manejo (tesis de Licenciatura en Ciencias de la tierra). Facultad de Ciencias, UNAM. México.

Escriche, M. M. (1999). Medio físico y turismo rural: una aportación para los informadores turísticos. Cuadernos de Turismo, (3), 93-II4. https://revistas.um.es/turismo/article/view/2297I

García-Romero A., y Muñoz J. (2002). El paisaje en el ámbito de la Geografía. Col. Temas Selectos de la Geografía de México, N.o III.2. Instituto de Geografía, UNAM. 
Gómez-Zotano, J., Riesco-Chueca, P., Frolova, M., y Rodríguez-Rodríguez, J. (2018). The landscape taxonomic pyramid (LTP): a multi-scale classification adapted to spatial planning. Landscape Research, 43(7), 984-999. h ttps://doi.org/10.1080/0I426397.2017.I40402I

Gordon, T. (1994). The Delphi Method.I En J. C. Glen y T. J. Gordon (Eds.), Futures Researcb Methodology (pp. I-33). American Council for The United Nation University, Millennium Project.

Gutiérrez-López, D. (2018). Evaluación de la calidad e impacto ambiental de los atractivos turísticos, a partir de la aplicación de indicadores del paisaje. Municipio Isidro Fabela, Estado de México (tesis de Licenciatura en Geografía). Facultad de Filosofía y Letras, UNAM. México.

Instituto de Información e Investigación Geográfica, Estadística y Catastral del Estado de México (Igecem) (20I6). Estadística Básica Municipal. Isidro Fabela. Gobierno de México, Igecem.

Instituto Nacional de Estadística, Geografía e Informática (Inegi). (1983). Carta de uso de suelo y vegetación. Escala 1.50,000. EI4A28 Villa del Carbón. Ciudad de México, Inegi.

Instituto Nacional de Estadística, Geografía e Informática (Inegi). (1999). Ortofotos digitales (esc. I:50,00o), claves: $E_{14} A_{28} 8$, EI4A28fy EI4A29d. Ciudad de México, Inegi.

Instituto Nacional de Estadística, Geografía e Informática (Inegi). (2010). Censo de Población y Vivienda 2010. Principales resultados por localidad (ITER). Gobierno de México, Inegi. https://datos.gob.mx/busca/dataset/c enso-de-poblacion-y-vivienda-20ro-principales-resultados-por-localidad-iter.

Jerez, O., y Serrano De La Cruz, M. A. (20I6). El interés didáctico de los paisajes alterados. La Reserva de la Biósfera de La Mancha Húmeda (España) como ejemplo de estudio. Contexto E。 Educaçao, 3I(99), 52-80. ht tps://doi.org/I0.21527/2179-1309.20I6.99.52-80

Kalivoda, O., Vojar, J., Skrivanová, Z., y Zahradník, D. (2014). Consensus in landscape preference judgments: The effects of landscape visual aesthetic quality and respondents' characteristics. Journal of Environmental Management, I37, 36-44. https://doi.org/10.1016/j.jenvman.20I4.02.009

Kenafsey, M. (200I). Rural cultural economy: tourism and social relations. Annals of Tourism Research, 28(3), 762-783. https://doi.org/10.1016/Sor60-7383(00)00077-3

Knudsen, D. C., Greer, C. E., Metro-Roland, M., y Soper, A. K. (Eds.). (2008). Landscape, Tourism and Meaning. Ashgate.

Knudsen, D. C., Metro-Roland, M. M., y Rickly-Boyd, J. M. (2013). Landscape studies and tourism research. En P. Howard, I. Thompson y E. Waterton (Eds.), The Routledge Companion to Landscape Studies (pp. 286-295). Routledge.

Lane, B., y Kastenholz, E. (2015). Rural tourism: the evolution of practice and research approaches - towards a new generation concept? Journal of Sustainable Tourism, 23(8-9), I133-1156. https://doi.org/10.1080/09669582.20 15.1083997

Mata, R. (20II). La gestión del paisaje. En M. Simancas y A. Cortina (Coords.), Retos y perspectivas de la gestión del paisaje en Canarias. Reflexiones en relación con el 10 o aniversario de la firma del Convenio Europeo del Paisaje (pp. 19-40). Tenerife, UIMP y Gobierno de Canarias.

Mateo, J. M. (2008). Paisajes naturales, Geografía de los paisajes. Primera Parte. Universitaria.

Mateos, M. R. (2016). Paisaje, patrimonio y turismo de surf: factores de atracción y motivación en el Parque Natural del Estrecho, España. Cuadernos de Turismo, (37), 351-376. https://doi.org/10.6018/turismo.37.256271

Mearns, K. F. (2012). Lessons from the application of sustainability indicators to community-based ecotourism ventures in Southern Africa. African Journal of Business Management, 6(26), 7851-7860. https://doi.org/10.5897 /AJBMir.258I 
Méndez-Méndez, A., Serrano De La Cruz, M. A., Salinas, E., y García-Romero, A. (2018). Propuesta metodológica basada en indicadores para la valoración del potencial turístico del paisaje en áreas rurales: el caso del municipio de Atlautla (México). Cuadernos de Turismo, (42), 335-354. https://doi.org/10.6018/turismo.42.15

Meng, F., y Uysal, M. (2008). Effects of Gender Differences on Perceptions of Destination Attributes, Motivations and travel Values: An Examination of a Nature - based Resort Destination. Journal of Sustainable Tourism, I6(4), 445-466. https://doi.org/10.1080/0966958080215423I

Mikulec, J., y Antoušková, M. (20II). Landscape and tourism potential in the protected landscape areas. Agricultural Economics, 57(6), 272-278. https://doi.org/10.17221/16/20II-AGRICECON

Muñoz, J. (1998). Paisaje y geosistema. Una aproximación desde la Geografía Física. En E. Martínez de Pisón (Dir.), Paisaje y Medio Ambiente (pp. 45-55). Fundación Duques de Soria y Universidad de Valladolid.

Nogué, J., De San Eugenio, J., y Sala, P. (2019). La implementación de indicadores de lo intangible para catalogar el paisaje percibido. El caso del Observatorio del Paisaje de Cataluña. Revista de Geografía Norte Grande, (72), 75-91. http://dx.doi.org/10.4067/S0718-34022019000100075

Nogué, J., Puigbert, L., y Bretcha, G. (Eds.). (2009). Indicadorsde paisatge. Reptes i perspectives. Observatorio del Paisaje de Cataluña, Obra Social de Caixa Catalunya.

Organization for Economic Co-operation and Development (OECD). (2003). OECD Environmental Indicators: Development, Measurement and Use. Paris, OECD.

Picazo, H. (2012). ¿Por qué viajamos donde viajamos? Una Introducción a la estrecha relación entre Turismo y Paisaje. Revista Eubacteria,(29), I-5. https://www.um.es/eubacteria/turismoypaisaje.pdf

Pié i Ninot, R., Rosa, C., Álvarez, I., y Nebot, N. (Coords.). (2019). Turismo y paisaje. Tirant lo Blanch.

Prat Forga, J. M., y Cànoves Valiente, G. (20I4). Integrated cultural tourism: new experiences in mountain areas. Tourismos. An International Multidisciplinary Journal of Tourism, 9(2), 15-32.

Rivera, M., y Rodríguez, L. (Coords.). (2012). Turismo responsable, sostenibilidad y desarrollo local comunitario. Cátedra Intercultural, Universidad de Córdoba.

Robín, C. F., Valencia, C., Santander, P., y Orrego, R. (2015). Turismo de intereses especiales: investigación de mercados sobre las motivaciones desde la perspectiva del cliente. Revista Internacional Administración y finanza, 8(I), 5-9. https://www.theibfr.com/wpfb-file/riaf-v8ni-20I5-4-pdf/

Salinas, E., Mirandola, P. H., De Souza, M. H., y Garcia, L. R. (2019). El estudio de los paisajes como fundamento para la evaluación del potencial turístico del municipio Paranaiba, Ms, Brasil. Gran Tour: Revista de Investigaciones Turísticas,(19), 79-102. https://eutm.es/grantour/index.php/grantour/article/view/9I

Salinas, E., y La O, J. A. (2006). Turismo y sustentabilidad: de la teoría a la práctica en Cuba. Cuadernos de Turismo, (17), 203-223. https://revistas.um.es/turismo/article/view/17931

Santos-Pavón, E., Fernández-Tabales, A., y Muñoz-Yules, O. (2016). La incorporación del paisaje a la planificación turística. Análisis de la estrategia de turismo sostenible de Andalucía. Cuadernos de Turismo, (37), 175-202. ht tps://doi.org/10.6018/turismo.37.25620I

Serrano, D. (2008). Ensayo metodológico para la valoración estética del paisaje. Aplicación en Muntanyes D’Ordal, Barcelona. Geographicalia, (54), 99-II2. https://doi.org/10.26754/ojs_geoph/geoph.2008541099

Simpson, E. H. (1949). Measurement of Diversity. Nature, (163), 688. https://doi.org/10.1038/163688ao

Tarroja, A. (2006). Transformaciones territoriales y valoración social del paisaje. En R. Mata y A. Tarroja (Coords.), El paisaje y la gestión del territorio: criterios paisajisticos en la ordenación del territorio y el urbanismo (pp. 4I-50). Diputación de Barcelona. 
Torres, R. (2003). Linkages between tourism and agriculture in México. Annals of Tourism Research, 30(3), 546-566. https://doi.org/10.1016/So160-7383(02)00103-2

Vallés, M., Galiana, F., y Bru, R. (2012). Towards Harmonisation in Landscape Unit Delineation: An Analysis of Spanish Case Studies. Landscape Research, 38(3), 329-346. https://doi.org/I0.I080/0I426397.20II.647896

Villegas-Martínez, D. (2016). Turismo rural como estrategia de desarrollo local en Isidro Fabela y la región de monte Alto, Estado de México (tesis de maestría en Agroindustria Rural, Desarrollo Rural y Turismo Agroalimentario). Universidad Autónoma del Estado de México. http://ri.uaemex.mx/handle/20.500.11799/65708

Villegas-Martínez, D., Gutiérrez Cedillo, J. G., y Juan Pérez, J. I. (2019). Estrategia de desarrollo local sustentable en el Área Natural Protegida Parque Otomí Mexica del Estado de México. Circuitos turísticos en el municipio de Isidro Fabela. Pasos. Revista de Turismo y Patrimonio Cultural, I7(4), 725-745. https://doi.org/10.25I45/j.paso S.2019.17.05I

Willson, G. B., Mcintosh, A. J., y Zahra, A. L. (2013). Tourism and Spirituality: A Phenomenological Analysis. Annals of Tourism Research, (42), 150-168. https://doi.org/10.1016/j.annals.2013.01.016

Wlodarczyk, B. (2009). The landscapes of tourism space. Tourism, I9(I-2), 83-90. https://doi.org/10.2478/Vioro6-० 09-00II-Z

Zuluaga, P. A. (2006). Una mirada al paisaje como recurso turístico. Revista Interamericana de Ambiente y Turismo, 2(I), 76-82. http://dx.doi.org/I0.4067/riatvol2issipp76-82\%250718-235X

\section{Notas}

* Artículo de investigación

Financiación: La investigación fue financiada por la Universidad Nacional Autónoma de México, a través del proyecto PAPIITIN30082I.

CC BY 